\title{
Liderança autêntica: uma revisão integrativa
}

\begin{abstract}
RESUMO
Liderança autêntica é uma das principais teorias de liderança surgidas nos últimos dez anos. Ela se propõe a construir organizações mais autênticas, com líderes mais autoconscientes e relações mais transparentes e éticas. Propôs-se aqui o desenvolvimento de uma revisão integrativa das publicações sobre o tema em periódicos indexados às bases de dados EBSCO, ISI Web of Knowledge, Scopus e SciElo, entre 2000 e 2013. Após as fases de busca e seleção da literatura, integraram a revisão 136 artigos. Foram constatadas três principais vertentes de estudos a respeito do tema: a primeira decorrente da gestão escolar; a segunda, da enfermagem; e a terceira, da gestão organizacional. Com o avanço das pesquisas sobre o tema, a visão da gestão organizacional acabou consolidandose, inclusive na área da educação e da enfermagem. Há uma preponderância de estudos teóricos acerca do tema, tendo, após o desenvolvimento do Questionário da Liderança Autêntica (ALQ), as pesquisas se direcionado mais à confirmação e validação dos construtos e do instrumento, do que ao aprofundamento da teoria. Assim, constatou-se que mais pesquisas ainda se fazem necessárias sobre o tema, recomendando-se o desenvolvimento de estudos empíricos qualitativos, inclusive no contexto brasileiro, onde foram encontrados apenas dois artigos científicos que abordam diretamente a matéria.
\end{abstract}

Palavras-chave: Liderança autêntica. Ética. Valores. Autenticidade. Capital psicológico. 


\section{INTRODUÇÃO}

A sociedade humana encontra-se em um período de transição, da sociedade industrial para a sociedade do conhecimento, com reflexos não somente na economia, mas também na forma como as pessoas vivem, relacionam-se, veem o propósito de suas vidas e, certamente, na maneira como se comportam no ambiente de trabalho (CASTELLS, 2002).

Nesse novo contexto, os modelos e soluções outrora válidos para as organizações da sociedade industrial podem não ser mais eficazes. Prova disso são os escândalos corporativos do início do século XXI e também a crise econômica iniciada em 2008, que abalou os Estados Unidos da América e que ainda assola a Europa. Eventos como esses indicam a necessidade de se pensar uma nova economia, novas organizações e, consequentemente, novas formas de liderança.

A liderança autêntica nasceu com a intenção de ajudar a resolver os problemas desse novo contexto. Seu foco é produzir relações humanas e organizações mais autênticas, com base no desenvolvimento de líderes mais conformes com seu próprio eu (self) e mais transparentes na forma de se relacionar com os outros (AVOLIO; GARDNER, 2005).

Para que se possa contribuir com as pesquisas e com o desenvolvimento de uma área do conhecimento científico, é elementar primeiramente se conhecer o estado da arte dos estudos sobre a temática. Com esse espírito, pretende-se neste artigo responder às seguintes questões: quais são os principais construtos abordados pelos pesquisadores da liderança autêntica? Quais são os principais métodos de pesquisa adotados? Quais são os principais resultados alcançados até o presente momento?

Para buscar a resposta a tais questionamentos, desenvolveu-se uma revisão integrativa dos artigos publicados a respeito de liderança autêntica entre 2000 e 2013 em periódicos indexados às bases de dados EBCO, ISI Web of Knowledge, Scopus e SciElo.

\subsection{Objetivo geral}

Analisar a literatura sobre liderança autêntica, para entender quais são os principais construtos adotados pelos seus pesquisadores, assim como os principais métodos de pesquisa e os principais resultados alcançados até o presente momento.

\subsection{Objetivos específicos}

a) Identificar as principais correntes de estudo da liderança autêntica.

b) Analisar as principais críticas encontradas na literatura à liderança autêntica.

c) Identificar as publicações brasileiras sobre o tema.

d) Analisar as publicações brasileiras acerca do tema.

\section{METODOLOGIA}

Propõe-se neste trabalho o desenvolvimento de uma revisão integrativa das publicações sobre liderança autêntica, tratando-se de uma espécie de revisão sistemática que permite abarcar o maior número e espécies de estudos que envolvem o objeto da pesquisa.

Existem diversos tipos de revisão da literatura, que podem ser congregados em duas categorias fundamentais: a revisão narrativa e a revisão sistemática. Revisões narrativas são publicações amplas, que buscam discutir o desenvolvimento de um determinado assunto sob o ponto de vista teórico ou contextual, em que não há a obrigatoriedade da apresentação das fontes de informação utilizadas, da metodologia para busca das referências, nem dos critérios utilizados na avaliação e seleção dos trabalhos, marcada pela interpretação, análise e crítica pessoal do autor (COOK; MULROW; HAYNES, 1997; ROTHER, 2007).

Já a revisão sistemática é desenvolvida com o objetivo de responder a um problema específico e utiliza-se de métodos explícitos e sistemáticos para identificar, selecionar e avaliar criticamente os estudos acerca da temática escolhida pelo autor, e para a coleta e análise de dados dos estudos incluídos na revisão (ROTHER, 2007).

A revisão sistemática pode ser subdividida, ainda, em quatro métodos de revisão específicos, cada um com um objetivo de análise e procedimentos metodológicos distintos, tratando-se: (a) da meta-análise, que combina os resultados de múltiplos estudos primários que desenvolveram pesquisas semelhantes, empregando métodos estatísticos para incrementar a objetividade das conclusões obtidas nesses estudos; (b) da revisão sistemática como técnica de pesquisa, que busca 
coletar todos os resultados empíricos que atendem a um critério preestabelecido para responder a determinada questão de pesquisa; (c) a revisão qualitativa, representada pelo conjunto de métodos para combinação e análise dos resultados de pesquisas qualitativas; (d) e ainda a revisão integrativa, uma categoria mais abrangente que as anteriores, podendo compreender a revisão da literatura teórica ou empírica, qualitativa ou quantitativa, a depender do propósito da pesquisa (BOTELHO; CUNHA; MACEDO, 2011; GREEN et al., 2011; WHITTEMORE, 2005; WHITTEMORE; KNAFL, 2005).

Dentre os diferentes métodos de revisão sistemática da literatura, optou-se neste trabalho pela revisão integrativa, pois tal modalidade permite o desenvolvimento de estudos de revisão com um escopo muito mais amplo do que as demais modalidades, integrando publicações de cunho teórico ou empírico, pautadas em abordagens quantitativas ou qualitativas.

A revisão integrativa permite resumir o passado da literatura, de modo que se alcance uma compreensão mais abrangente de um fenômeno em particular, apresentando-se o estado da técnica e permitindo a contribuição ao desenvolvimento teórico da área estudada com a geração de novos conhecimentos, assim como com implicações para as práticas organizacionais (BOTELHO; CUNHA; MACEDO, 2011; MENDES; SILVEIRA; GALVÃO, 2008; WHITTEMORE; KNAFL, 2005).

No entanto, por permitir o desenvolvimento de estudos com uma maior abrangência de estudos científicos, a literatura destaca que os riscos da revisão integrativa são maiores do que as demais modalidades de revisão, por causa especialmente da complexidade de analisar e sintetizar conjuntamente essas diferentes fontes (JACKSON, 1980; WHITTEMORE, 2005; WHITTEMORE; KNAFL, 2005). Desse modo, é fundamental a implementação de estratégias de análise que garantam a confiabilidade da revisão desenvolvida pelo pesquisador.

Tendo em vista a importância do cumprimento rigoroso das fases da revisão integrativa, para assim diminuir os riscos da pesquisa, adotou-se neste trabalho a proposta de Botelho, Cunha e Macedo (2011), que consideram ser a revisão integrativa composta pelas seguintes seis fases, representadas na Figura 1:

1) identificação do tema e seleção da questão de pesquisa;

2) estabelecimento dos critérios de inclusão e exclusão;

3) identificação dos estudos pré-selecionados e selecionados;

4) caracterização dos estudos selecionados;

5) análise e interpretação dos resultados;

6) apresentação da revisão.

Figura 1 - Processo de revisão integrativa

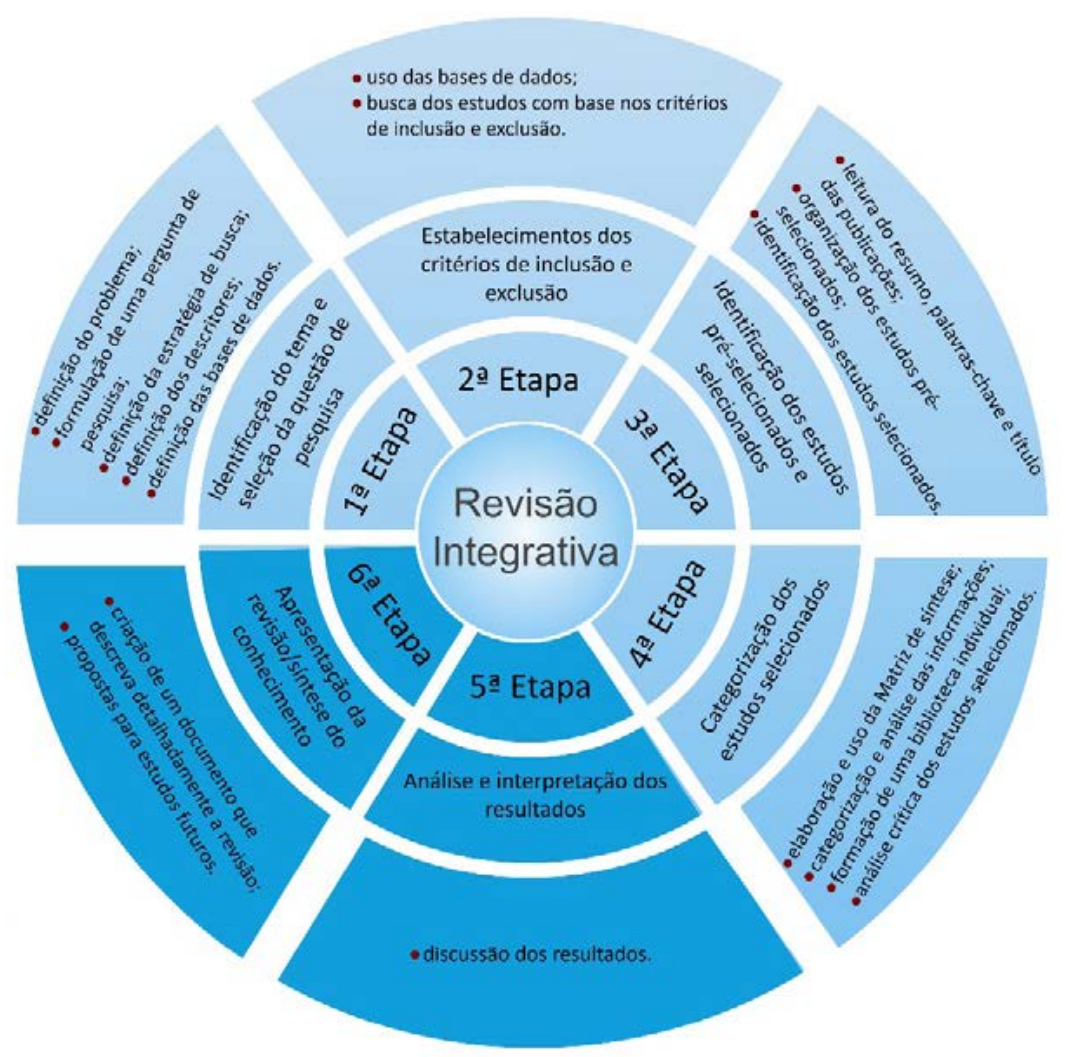

Fonte: Botelho, Cunha e Macedo (2011, p. 129) 
O modelo de revisão integrativa proposto pelos autores pode ser considerado como confiável, tendo em vista que estrutura similar pode ser encontrada em outras propostas de revisão integrativa, como no trabalho de Souza, Silva e Carvalho (2010), e também no de Whittemore e Knafl (2005).

\section{TEORIA DA LIDERANÇA AUTÊNTICA}

A teoria da liderança autêntica nasce com a proposta de explicar melhor a pessoa do líder e da forma como se estabelece a relação entre este e seus liderados sob a perspectiva de relações mais positivas. Como forma de identificar o espírito do líder autêntico, Sparrowe (2005, p. 420) cita as palavras de Shakespeare em sua obra Hamlet, em que o personagem Polonius fala a Laertes para"ser quem realmente se é".

Na teoria da liderança autêntica, a concepção de autenticidade origina-se na psicologia positiva, que trata autenticidade como ser, ao mesmo tempo, dono dos seus próprios pensamentos e crenças, e agir de modo que seja correspondente ao próprio eu. Nesse sentido, conforme Gardner e Schermerhorn (2004, p. 271), ser autêntico é "dizer o que você realmente pensa, sentir e se comportar de acordo".

A liderança autêntica tem por fundamento a aplicação dos conceitos da psicologia positiva às relações de liderança. Ao contrário das abordagens tradicionais da psicologia, que trabalham o ser humano com base em suas fraquezas e desvios, a psicologia positiva visa reforçar os pontos fortes, por meio do estudo das emoções positivas, dos traços de personalidade e habilidades positivos e das instituições humanas positivas (SELIGMAN, 2004).

Assim, baseado na psicologia positiva, Luthans (2002) propôs o conceito de comportamento organizacional positivo (Positive Organizational Behavior ou POB), considerado como a forma pela qual os pontos fortes e as capacidades psicológicas dos indivíduos nas organizações podem ser mensurados, desenvolvidos e eficazmente geridos para a melhoria da performance no ambiente de trabalho (GARDNER; SCHERMERHORN, 2004).

Os principais estados indicativos do comportamento organizacional positivo são confiança, esperança, otimismo e resiliência, que são agregados na categoria "capital psicológico" (LUTHANS; AVOLIO, 2003).

Da concepção de comportamento organizacional positivo, decorre a ideia de liderança autêntica, como o tipo de relação, dentro da organização, que promove esse modelo comportamental. Avolio et al. (2004, p. 802) definem como líderes autênticos “[...] pessoas que alcançaram altos níveis de autenticidade, que sabem quem são, no que acreditam e valorizam, e que agem baseados nesses valores e crenças enquanto interagem de modo transparente com os outros".

Apesar de manter estreita relação com a proposta de Avolio et al. (2004), a definição de Walumbwa et al. (2008) enfatiza a liderança autêntica como um padrão de comportamento do líder.

Especificamente, definimos liderança autêntica como um padrão de comportamento do líder que se baseia e promove ambos, as capacidades psicológicas e um clima ético positivo, para fomentar uma maior autoconsciência, uma perspectiva moral internalizada, o processamento balanceado de informações e a transparência relacional por parte de líderes que trabalham com seguidores, promovendo o autodesenvolvimento positivo.

Gardner et al. (2005) ressaltam que o líder não se autentica apenas tendo em vista uma necessidade interna, pois a liderança envolve uma relação intersubjetiva. Assim sendo, o líder autêntico promove autenticidade juntamente com seus liderados, estabelecendo-se, desse modo, relações mais autênticas. $O$ próprio liderado, como ser humano autêntico, deve contribuir para o desenvolvimento do melhor de seu líder, o que é considerado por Shamir e Eilam (2005) como uma relação de seguir autêntico (authentic followership). Nesse processo, tornar-se-ia possível ao final tornar as próprias organizações mais autênticas.

Uma das grandes questões da liderança autêntica é se diferenciar de outras teorias de liderança, como as chamadas lideranças inspiracionais, como a carismática e a transformacional, ou ainda a liderança ética, a liderança servidora e a liderança espiritual. A esse respeito, Avolio e Gardner (2005) propõem a liderança autêntica como um construto-raiz, considerando que a proposta abarca todas as formas positivas de liderança; diferencia-se, porém, delas por estar ancorada no senso mais profundo do próprio eu do líder, sendo tais líderes autoconscientes, conhecedores de sua relação com a vida em geral, valores e crenças. Aliás, a ética do líder não é uma ética externalizada, trata-se das próprias concepções de certo ou errado, bom ou mau, justo ou injusto, o que é chamado pelos autores de perspectiva moral internalizada (AVOLIO; GARDNER, 2005).

A liderança autêntica é uma teoria que não busca descrever como é o líder, com base na observação da realidade. Trata-se de uma teoria normativa, que propõe como deve ser e agir o líder, 
com base nos princípios da Psicologia Positiva e no comportamento organizacional positivo. Nesse sentido, ao contrário de outras abordagens da liderança, o desafio da teoria da liderança autêntica é demonstrar, de maneira empírica, a validade de sua proposta. A seguir, serão apresentados os procedimentos adotados na presente revisão integrativa.

\section{DESENVOLVIMENTO DA REVISÃO INTEGRATIVA}

Seguindo a estrutura proposta por Botelho, Cunha e Macedo (2011), na sequência são apresentadas as quatro primeiras fases de desenvolvimento da revisão integrativa.

Após o estabelecimento do tema de pesquisa, a primeira decisão tomada foi a definição do problema de pesquisa, sendo estabelecidas duas questões: (1) Há uma única teoria da liderança autêntica, ou esta é constituída por proposições distintas? (2) Há uma predominância de estudos teóricos sobre liderança autêntica, ou o número de estudos empíricos é igualmente relevante?

Foram então pesquisadas as bases de dados escolhidas, a EBSCO, ISI Web of Knowledge, SciElo e Scopus, considerando-se que todas elas possuem notório reconhecimento nacional e internacional, bem como contemplam grande parte dos principais periódicos científicos na área de liderança. A estratégia de busca adotada foram artigos científicos publicados entre 2000 e 2013, na língua inglesa. O descritor utilizado para a busca foi authentic* AND leader*, para que fossem encontradas publicações com ambas as palavras, e o asterisco permitindo a localização de qualquer sufixo.

Em seguida, foram definidos os seguintes critérios de inclusão e exclusão: o artigo deve tratar sobre liderança autêntica, aparecendo o referido termo no título, no resumo, ou nas palavras-chave; o artigo deve abordar diretamente a liderança autêntica, não bastando o aparecimento das palavras "liderança","líder", "autêntico(a)" e"autenticidade" de maneira esparsa no texto; somente serão aceitos artigos científicos publicados em periódicos científicos; o texto completo deve estar disponível para acesso.

A pesquisa nas bases de dados retornou 1.700 artigos científicos. Em seguida, prosseguiu-se à pré-seleção dos artigos e, com base nos critérios definidos, foram obtidos 177 artigos.

Então, foram baixados os textos dos artigos na íntegra, para a leitura e seleção. Nessa fase, foram excluídos textos que, muito embora fizessem menção aos descritores, não trabalhavam diretamente com a liderança autêntica; além de textos que não são artigos científicos publicados em periódicos. Dessa maneira, foram excluídos 41 trabalhos, restando um total de 136 artigos para a revisão.

Por fim, na quarta fase, os artigos foram lidos na íntegra, considerando os seguintes elementos: objetivos; tipo de estudo (teórico ou empírico, quantitativo ou qualitativo); variáveis ou construtos adotados no trabalho; contexto de realização do estudo (se empírico); principais resultados; temas ou recomendações para pesquisas futuras.

A partir da coleta dessas informações dos artigos estudados é que se desenvolveu a análise crítica da produção científica sobre liderança autêntica, que será apresentada na seção a seguir.

\section{ANÁLISE E INTERPRETAÇÃO DOS RESULTADOS}

O estudo da literatura sobre liderança autêntica permitiu importantes constatações quanto às origens e à definição do construto, às fases de desenvolvimento das pesquisas acerca do tema, assim como das principais críticas dirigidas a esta proposta, conforme se verá a seguir.

A leitura e a análise dos artigos selecionados permitiram a identificação de três matrizes de discussão acerca da liderança autêntica: uma vinculada à área da gestão educacional, outra à área da gestão de negócios e uma terceira ligada à enfermagem.

A liderança autêntica começou a ser estudada sob a perspectiva da gestão educacional na década de 1980. Sua preocupação maior é a construção da autenticidade do líder escolar, incorporando-se na visão de autenticidade todas as qualidades positivas de um líder. Conforme Begley e Stefkovich (2007, p. 570) liderança autêntica é "[...] uma metáfora para práticas profissionalmente eficazes, eticamente baseadas e conscientemente reflexivas na administração educacional. É a liderança baseada no conhecimento, informada pelos valores e habilidosamente executada".

$\mathrm{Na}$ área da enfermagem, a liderança autêntica surge muito menos como uma proposta científica e mais como um slogan, sobre o qual posteriormente foram construindo-se fundamentos teóricos. Conforme Shirey (2006, p. 257), em 2001 a Associação Americana de Enfermeiras (AACN) formulou um termo de compromisso para promover a criação de ambientes de trabalho mais saudáveis, humanos e respeitosos com os familiares dos pacientes e com as enfermeiras, estabelecendo uma lista de padrões a serem buscados, dentre os quais estava a liderança autêntica, definida como "[...] 
a cola que une um ambiente de trabalho saudável".

$\mathrm{Na}$ área da gestão, o debate sobre a liderança autêntica primeiramente surgiu entre os práticos da gestão (practicioners), para posteriormente ganhar espaço na discussão acadêmica. A obra de Terry (1993) pode ser considerada a primeira a trabalhar com o construto. Dez anos depois, George (2003), ex-presidente da Medtronic, publicou um livro intitulado Liderança Autêntica, que ganhou notoriedade e serviu de estímulo à publicação científica a respeito do tema.

Além das publicações dos práticos, a análise dos textos permitiu identificar que a teoria da liderança autêntica nasceu dentro da teoria da liderança transformacional, especialmente inspirada no artigo de Bass e Steidlmeier (1999). Esse artigo adota uma perspectiva ética e divide as duas categorias: (1) os líderes transformacionais autênticos, líderes que exercem a liderança pautada em princípios éticos; e (2) os líderes transformacionais inautênticos, aqueles que não agem pautados em valores éticos, mas, pelo contrário, utilizam-se da sua capacidade de influência para controlar e conformar os liderados aos seus interesses.

Dentre os artigos estudados, especialmente as publicações de Price (2003), Jingping (2004) e Driscoll e McKee (2007) são representativas dessa transição da liderança transformacional para a liderança autêntica, analisando a liderança transformacional com base em princípios éticos.

Outra evidência dessa relação de continuidade entre a liderança transformacional e autêntica é o histórico das pesquisas de dois dos principais pesquisadores da liderança autêntica, Avolio e Walumbwa, que anteriormente haviam publicado diversos trabalhos sobre liderança transformacional, inclusive com Bass, autor de uma das principais obras dessa abordagem Leadership and performance beyond expectation, de 1985.

Em 2003, Luthans e Avolio publicaram um capítulo de livro sobre liderança autêntica e passaram a publicar artigos científicos a propósito do tema, culminando em um evento realizado em 2004, na Universidade de Nebraska-Lincoln, cujos principais trabalhos foram selecionados para uma edição especial do periódico Leadership Quarterly, lançado em 2005 (AVOLIO; GARDNER, 2005).

Essa edição especial serviu de paradigma para grande parte das pesquisas realizadas posteriormente. Sobretudo os artigos de Avolio e Gardner (2005); Gardner et al. (2005); Ilies, Morgeson e Nahrgang (2005); e Mitchie e Gooty (2005) exerceram marcante influência nos anos seguintes, especialmente até 2008.

No período de 2004 a 2008, o foco era teórico, afirmando os valores do capital psicológico, do comportamento organizacional positivo e da ética do líder como indicadores de um ambiente organizacional mais autêntico. Dos 34 artigos selecionados desse período, 26 deles apresentam estudos de cunho teórico e oito estudos são empíricos.

Referidos estudos não têm por objeto direto validar a teoria, mas demonstrar a relevância de certas características para a construção do líder autêntico ou possível correspondência da teoria com a realidade. Na linha dos estudos quantitativos, estão trabalhos como o de Branson (2007), que trata da necessidade do autoconhecimento como base para o desenvolvimento da liderança autêntica em diretores de escolas; o trabalho de Tate (2008), a respeito da relação do automonitoramento com liderança autêntica; e ainda os de Jensen e Luthans (2006a, 2006b), que propõem o estudo de empreendedores como líderes autênticos. Na linha dos estudos qualitativos, destacam-se os trabalhos de Endrissat, Müller e Kaudela-Baum (2007), com líderes experientes da Suíça, e o de Turner e Mavin (2008), que desenvolve estudo semelhante nos Estados Unidos da América.

Foi possível constatar que houve uma grande mudança nos rumos das pesquisas sobre liderança autêntica com a publicação de um artigo de Walumbwa et al. (2008). Esse artigo buscou responder à provocação lançada por um dos artigos publicados na edição especial da Leadership Quarterly, de Cooper, Scandura e Schriesheim (2005), que questionava a necessidade de uma nova abordagem da liderança, tendo em vista as demais já existentes; e também a falta de validação científica dos principais construtos defendidos pela teoria.

Segundo Cooper, Scandura e Schriesheim (2005), antes de se desenvolver estratégias para o desenvolvimento de liderança autêntica, os pesquisadores da área necessitam considerar cuidadosamente quatro pontos críticos:

1) definir e mensurar o construto;

2) determinar a validade discriminante do construto;

3) identificar resultados relevantes do construto (testar a rede nomológica do construto);

4) confirmar se a liderança autêntica pode ser ensinada.

A resposta dada por Walumbwa et al. (2008) ocorreu na forma de um instrumento de mensuração da liderança autêntica, o Questionário da Liderança Autêntica (em inglês Authentic Leadership Questionnaire - ALQ) - um questionário de dezesseis fatores que avalia as principais características atribuídas à liderança autêntica: autoconsciência, transparência relacional, perspectiva moral internalizada e processamento balanceado. Os autores utilizaram-se de cinco amostras diversas 
obtidas nos Estados Unidos da América, no Quênia e na China.

Posteriormente à disponibilização do ALQ como instrumento de mensuração da liderança autêntica, o foco das pesquisas se alterou, da busca pela fundamentação teórica adequada à proposta da liderança autêntica, para a validação empírica, especialmente quantitativa, do construto. Nesse movimento, engajaram-se tanto novos pesquisadores, como Caza et al. (2010), na Nova Zelândia; e Zheng et al. (2011), na China; quanto os pesquisadores precursores do debate, como Gardner, Fischer e Hunt (2010); Walumbwa et al. (2010) e Hannah, Walumbwa e Fry (2011).

A partir de 2008, ocorreu a consolidação da teoria da liderança autêntica proveniente da Universidade de Nebraska-Lincoln como norte para as discussões sobre o tema, inclusive para a área da educação e para a enfermagem. Na discussão sobre os líderes educadores, trabalhos como o de Chuang e Bird (2011) pautaram-se diretamente nessa visão de liderança autêntica oriunda da área da gestão. O mesmo se pode dizer do artigo de Rahman, Abiodullah e Quraishi (2010), sobre o perfil de liderança autêntica na área da educação para a promoção de escolas mais democráticas no Paquistão.

O artigo de Walumbwa et al. (2008) impactou, também, na área de enfermagem, com destaque para as pesquisas desenvolvidas na Universidade de Western Ontario, no Canadá, como se encontra em Wong e Cummings (2009a, 2009b), Wong, Laschinger e Cummings (2010), Giallonardo, Wong e Iwasiw (2010), Wong e Giallonardo (2013), dentre outros.

Os rumos das pesquisas fazem parecer que a liderança autêntica seja uma teoria que já está encaminhando-se para sua maturidade e afirmando-se como um todo concentrado nas características já apresentadas. Aliás, o fato de que poucas contribuições no nível teórico foram dadas por pesquisadores de outras nações, que não dos Estados Unidos da América, parece reforçar a consolidação do modelo de liderança autêntica proposto pelo principal grupo de pesquisadores.

Este é o grande receio de Humphreys et al. (2011a, 2011b), que veem como elementar que ainda se questione e pesquise o verdadeiro significado da autenticidade do líder. Nesse sentido, foram encontradas na literatura outras propostas de liderança autêntica, propostas de outros instrumentos de análise empírica do construto, assim como críticas dirigidas à vertente da liderança autêntica nascida na Universidade de Nebraska-Lincoln.

Propostas alternativas podem ser encontradas nos seguintes autores: Sparrowe (2005), que propõe uma fundamentação filosófica à liderança autêntica, com base na teoria do eu-narrativo de Ricoeur, considerando que a autenticidade não é um processo marcado pelo autoconhecimento, como processo individual. De acordo com o autor, o conhecimento de si próprio se dá na relação entre as pessoas. Shamir e Eilam (2005), que consideram a liderança autêntica como uma posição pessoal, não um estilo de liderança, ponderando que os outros tipos de líderes podem ser autênticos ou não. Novicevic et al. (2005, 2006), que buscam fundamentar a liderança autêntica nas proposições de Barnard, teórico da área da administração dos anos 1920-1950. Humphreys et al. (2011a), que encontram na história da fundadora de uma gravadora independente dos Estados Unidos da América, Lilian McMurry, um exemplo de líder autêntica, com base na qual tecem críticas à imagem de liderança autêntica da corrente dominante; e ainda em Humphreys et al. (2011b), que buscam na filosofia de Xenofonte, também, fundamentos para a liderança autêntica.

Do ponto de vista empírico, Neider e Schriesheim (2011) propõem um novo instrumento de avaliação da liderança autêntica, o Inventário da Liderança Autêntica (Authentic Leadership Inventory - ALI), e buscam demonstrar a maior confiabilidade do seu instrumento de avaliação, em comparação com o ALQ.

Seguindo uma linha mais crítica, Yammarino et al. (2008) consideram que o foco da liderança autêntica tem se dirigido quase que exclusivamente à perspectiva individual e à perspectiva da díade líder-liderado. Assim, os autores recomendam o desenvolvimento de estudos nos níveis meso e multi das organizações, considerando necessário o desenvolvimento de estudos igualmente nos níveis do grupo e da organização, para se afirmar a validade da proposta da liderança autêntica.

No entanto, as críticas mais contundentes à ideia da liderança autêntica estão nos trabalhos de Gardiner (2011) e de Ford e Harding (2011). O primeiro artigo acusa os teóricos da liderança autêntica de desconsiderarem o modo pelo qual as circunstâncias sociais e históricas afetam a habilidade de uma pessoa de ser um líder, bem como critica o uso do termo autenticidade, com base nos conceitos de unidade de Hannah Arendt e de autenticidade como resolutividade de Heidegger. O segundo artigo tem um tom mais crítico, a começar pelo título: "A impossibilidade do 'eu-verdadeiro' da liderança autêntica".

As críticas de Ford e Harding (2011) concentram-se nos seguintes pontos:

1) o conceito de eu-verdadeiro (true self), entendido como o autoconceito do líder autêntico, não condiz com a realidade, pois ignora as imperfeições dos indivíduos e promove uma identidade muito mais coletiva (da organização), do que propriamente individual; 
2) a liderança autêntica pode conduzir a uma dinâmica destrutiva dentro da organização;

3) a liderança autêntica é vista como mais uma abordagem que marca a relação de desigualdade entre líder e liderado, uma relação entre dominante e dominado.

Muito embora o tom crítico dado à teoria, especialmente por Ford e Harding (2011), seja excessivamente pessimista, constatou-se a importância de tais críticas à teoria da liderança autêntica. Deve-se considerar que essa nova proposta passou muitos anos incólume, sem críticas contundentes, para além da falta de validação empírica da teoria.

Além disso, as críticas também se dirigem à finalidade da teoria, que se diz ética e promotora de um comportamento organizacional mais positivo, mas que na prática de modo contraditório, e até mesmo paradoxal, pode servir de fundamentação para o mais inautêntico e dominante líder de todos, que saberá consolidar o seu controle sobre os liderados não mais apenas sobre a expectativa de altas remunerações, pelo status de se trabalhar com tal líder ou, ainda, por seu carisma, mas também pela habilidade de lidar com a dimensão da espiritualidade, emocionalidade e com a identificação do liderado com seu líder e com a organização.

Finalmente, apenas dois artigos científicos brasileiros foram encontrados, que atendiam aos critérios de seleção desta revisão: os trabalhos de Lanzoni e Meirelles (2011), e de Sobral e Gimba (2012). O primeiro trabalho é uma revisão integrativa da literatura da área de enfermagem sobre liderança, em que as autoras encontraram o conceito de liderança autêntica da AACN, apresentado neste artigo. Já o trabalho de Sobral e Gimba (2012) é um estudo empírico, de cunho quantitativo, que busca relacionar a teoria motivacional de valores humanos com a liderança autêntica. Essa pesquisa aplicou o ALQ no Brasil, muito embora os autores reconheçam a inexistência de estudos validando o instrumento para o contexto brasileiro.

\section{CONCLUSÃO}

A proposta deste trabalho foi o desenvolvimento de uma revisão integrativa das publicações lançadas em periódicos indexados a quatro bases de dados altamente reconhecidas no meio científico: EBSCO, ISI Web of Knowledge, SciElo e Scopus.

Com base no modelo de revisão integrativa proposto por Botelho, Cunha e Macedo (2011), foram selecionados 136 artigos científicos que atendiam aos critérios propostos e representam o estado da arte das pesquisas na área de liderança autêntica.

Analisada a literatura, constatou-se a aparência de maturidade das pesquisas em liderança autêntica, a indicar que esta seria mais uma abordagem consolidada da liderança. Entretanto, a conclusão desta revisão integrativa é outra, ainda há muito a se pesquisar, de maneira crítica e rigorosa, a respeito da liderança autêntica e do líder autêntico.

A proposta da liderança autêntica visa preencher a lacuna de outras tantas teorias e abordagens existentes, a consideração da importância e da influência do autoconhecimento e do estabelecimento de relações de liderança mais transparentes nas organizações. Porém, querer encerrar uma teoria como essa em um formato normativo traz consigo o risco de ser esta apenas mais uma boa ideia para a gestão organizacional, que no momento da sua operacionalização revela-se como um fenômeno bastante perigoso.

É preciso, portanto, mais estudos na área, inclusive das propostas alternativas de definição da liderança autêntica identificadas na literatura, até porque as críticas de Ford e Harding (2011) apontam para o risco de se conceder o título de autênticos a líderes e a organizações que não correspondem realmente aos princípios e valores da liderança autêntica, criando-se ambientes que, em vez de promover a individualidade dos liderados, a suprimem e a modelam aos valores e interesses individuais do líder e da organização.

Essa consequência não é um resultado determinístico, mas deve ser considerada como um risco que não foi objeto de suficiente consideração nos artigos estudados nesta revisão.

Outro aspecto percebido nesta revisão é o fato de a maioria dos trabalhos sobre liderança autêntica possuir um modelo de pesquisa teórico, ou, quando empírico, de bases quantitativas, sendo encontrados poucos estudos da realidade sob uma ótica qualitativa. Entende-se que essa é uma fraqueza da teoria, pois, muito embora os resultados de vários dos trabalhos empíricos tenham sido estatisticamente relevantes, há uma carência da coleta de dados vivenciais daqueles que estão em contexto de relação com líderes autênticos.

Assim, futuras pesquisas sobre liderança autêntica deveriam estudar o fenômeno sob bases qualitativas, desenvolvendo estudos como a etnografia, uma pesquisa fenomenológica, estudos de caso, dentre outras possibilidades.

Um último aspecto a ser destacado é a existência de poucos estudos sobre a liderança autêntica em outras nações, fora dos Estados Unidos da América, e especialmente no Brasil, o que indica o espaço aberto ao desenvolvimento de novas pesquisas sobre o tema. 


\title{
AUTHENTIC LEADERSHIP: AN INTEGRATIVE REVIEW
}

\begin{abstract}
Authentic leadership is one of the main theories of leadership which emerged in the last 10 years. It intends to develop more organizations that are authentic with leaders who are self-conscious and relations which are more transparent and ethical. An integrative review of publications on the topic in indexed journals to databases EBSCO, ISI Web of Knowledge, Scopus and SciELO, from 2000 until the end of 2013 was proposed. Following the stages of search and selection of literature, 136 articles were reviewed. Three main streams of studies on the topic where noted: the first arouse from school management; the second from nursing; and the third from organizational studies. With the advancement of the studies on the topic, the view from organizational management was consolidated, not only in organizational studies, but also in education and nursing. There is a preponderance of theoretical studies on the subject. After the development of the Authentic Leadership Questionnaire (ALQ) research was directed more to confirm and validate authentic leadership constructs and instrument rather than to further develop the theory. Thus, it was found that further studies are needed on the subject. The development of more qualitative empirical studies is recommended, including in the Brazilian context, where only two scientific articles addressing directly the issue were found.
\end{abstract}

Keywords: Authentic leadership. Ethics. Values. Authenticity. Psychological capital.

\section{REFERÊNCIAS}

AVOLIO, B. J.; GARDNER, W. L. Authentic leadership development: getting to the root of positive forms of leadership. Leadership quarterly, Amsterdam, v. 16, n. 3, p. 315-338, 2005. Disponível em: <http://search.ebscohost.com>. Acesso em: 15 jan. 2014.

AVOLIO et al. Unlocking the mask: a look at the process by which authentic leaders impact follower attitudes and behaviors. Leadership quarterly, Amsterdam, v. 15, n. 6, p. 801-823, 2004. Disponível em: <http://www.sciencedirect.com/>. Acesso em: 15 jan. 2014.

BASS, B. M.; STEIDLMEIER, P. Ethics, character, and authentic transformational leadership behavior. The leadership quarterly, Amsterdam, v. 10, n. 2, p. 181-217, Summer 1999. Disponível em: <www. sciencedirect.com/science/article>. Acesso em: 11 nov. 2013.

BEGLEY, P. T.; STEFKOVICH, J. Integrating values and ethics into post secondary teaching for leadership development: principles, concepts, and strategies. Journal of educational administration, Bingley, v. 45, n. 4, p. 398-412, 2007. Disponível em: <http://www.scopus.com/>. Acesso em: 18 dez. 2013.

BOTELHO, L. L. R.; CUNHA, C. J. C. A.; MACEDO, M. O método da revisão integrativa nos estudos organizacionais. Revista eletrônica gestão e sociedade, Belo Horizonte, v. 5, n. 11, p. 121-136, maio/ago. 2011. Disponível em: <http://www.gestaoesociedade.org/gestaoesociedade/article/ view/1220/906>. Acesso em: 6 dez. 2013.

BRANSON, C. Effects of structured self-reflection on the development of authentic leadership practices among Queensland primary school principals. Educational management

administration and leadership, Nottingham, v. 35, n. 2, p. 225-246, 2007. Disponível em: <http:// www.scopus.com/>. Acesso em: 18 dez. 2013.

CASTELLS, M. A sociedade em rede. 6. ed. rev. e ampl. Rio de Janeiro: Paz e Terra, 2002.

CAZA, A. et al. Psychological capital and authentic leadership: Measurement, gender, and cultural extension. Asia-Pacific Journal of Business Administration, Bingley, v. 2, n. 1, p. 53-70, 2010. Disponível em: <http://www.scopus.com/>. Acesso em: 18 dez. 2013.

CHUANG, W.; BIRD, J. J. Multi-level modeling of principal authenticity and teachers' trust and engagement. Academy of educational leadership journal, Candler, v. 15, n. 4, p. 125-147, 2011. 
Disponível em: <http://search.ebscohost.com>. Acesso em: 18 dez. 2013.

COOK, D. J.; MULROW, C. D.; HAYNES, B. H. Systematic reviews: synthesis of best evidence for clinical decisions. Annals of internal medicine, Philadelphia, v. 126, n. 5, p. 376-380, 1997. Disponível em: <http://search.ebscohost.com>. Acesso em: 19 nov. 2014.

COOPER, C. D.; SCANDURA, T. A.; SCHRIESHEIM, C. A. Looking forward but learning from our past: potential challenges to developing authentic leadership theory and authentic leaders. The leadership quarterly, Amsterdam, v. 16, n. 3, p. 475-493, 2005. Disponível em: <www. sciencedirect.com/science/article>. Acesso em: 18 dez. 2013.

DRISCOLL, C.; MCKEE, M. Restorying a culture of ethical and spiritual values: a role for leader storytelling. Journal of business ethics, Dordrecht, v. 73, n. 2, p. 205-217, 2007. Disponível em: <apps.webofknowledge.com>. Acesso em: 18 dez. 2013.

ENDRISSAT, N.; MÜLLER, W. R.; KAUDELA-BAUM, S. En route to an empirically-based understanding of authentic leadership. European management journal, Amsterdam, v. 25, n. 3, p. 207-220, 2007. Disponível em: <http://www.scopus.com/>. Acesso em: 18 dez. 2013.

FORD, J.; HARDING, N. The impossibility of the 'true self' of authentic leadership. Leadership, London, v. 7, n. 4, p. 463-479, Nov. 2011. Disponível em: <http://lea.sagepub.com/content/7/4/463. full.pdf+html>. Acesso em: 18 dez. 2013.

GARDINER, R. A. A critique of the discourse of authentic leadership. International journal of business \& social science, Radford, v. 2, n. 15, p. 99-104, 2011. Disponível em: <http://search. ebscohost.com>. Acesso em: 18 dez. 2013.

GARDNER, W. L. et al. "Can you see the real me?" A self-based model of authentic leader and follower development. The leadership quarterly, Amsterdam, v. 16, n. 3, p. 343-372, 2005. Disponível em: <www.sciencedirect.com/science/article>. Acesso em: 18 dez. 2013.

GARDNER, W. L.; FISCHER, D.; HUNT, J. G. Emotional labor and leadership: a threat to authenticity? The leadership quarterly, Amsterdam, v. 20, n. 3, p. 466-482, 2010. Disponível em: <www. sciencedirect.com/science/article>. Acesso em: 18 dez. 2013.

GARDNER, W. L.; SCHERMERHORN, J. R. Unleashing individual potential: performance gains through positive organizational behavior and authentic leadership. Organizational dynamics, Amsterdam, v. 33, n. 3, p. 270-281, 2004. Disponível em: <www.sciencedirect.com/science/article>. Acesso em: 18 dez. 2013.

GEORGE, B. Authentic leadership: rediscovering the secrets to creating lasting value. San Francisco: Josey-Bass, 2003.

GIALLONARDO, L. M.; WONG, C. A.; IWASIW, C. L. Authentic leadership of preceptors: predictor of new graduate nurses' work engagement and job satisfaction. Journal of nursing management, Chichester, v. 18, n. 8, p. 993-1003, 2010. Disponível em: <http://onlinelibrary.wiley.com/ doi/10.1111/j.1365-2834.2010.01126.x/pdf>. Acesso em: 18 dez. 2013.

GREEN, S. et al. Introduction. In: HIGGINS, J. P. T.; GREEN, S. (Ed.). Cochrane handbook for systematic reviews of interventions. London: The Cochrane Collaboration, (2011) cap. 1. Disponível em: <http://handbook.cochrane.org/>. Acesso em: 17 nov. 2014.

HANNAH, S. T.; WALUMBWA, F. O.; FRY, L. W. Leadership in action teams: team leader and members' authenticity, authenticity strength, and team outcomes. Personell psychology, Oxford, v. 64, n. 3, p. 771-802, 2011. Disponível em: <http://onlinelibrary.wiley.com/doi/10.1111/j.17446570.2011.01225.x/pdf>. Acesso em: 18 dez. 2013.

HUMPHREYS, J. H. et al. Lillian McMurry of trumpet records: integrity and authenticity in the 
charismatic, constructive narcissist leader. Journal of leadership and organizational studies, London, v. 18, n. 1, p. 40-55, 2011a. Disponível em: <http://www.scopus.com/>. Acesso em: 18 dez. 2013.

HUMPHREYS, J. H. et al. Towards the augmenting role of authenticity: Xenophon as leadership theorist. Management and organizational history, Milan, v. 6, n. 2, p. 183-207, 2011 b. Disponível em: <http://www.scopus.com/>. Acesso em: 18 dez. 2013.

ILIES, R.; MORGESON, F. P.; NAHRGANG, J. D. Authentic leadership and eudaemonic well-being: understanding leader-follower outcomes. Leadership quarterly, Amsterdam, v. 16, n. 3, p. 373394, 2005. Disponível em: <www.sciencedirect.com/science/article>. Acesso em: 18 dez. 2013.

JACKSON, G. B. Methods for integrative reviews. Review of educational research, London, v. 50, n. 3, p. 438-460, Fall 1980. Disponível em: <rer.sagepub.com/content/50/3/438.full.pdf + html >. Acesso em: 19 nov. 2014.

JENSEN, S. M.; LUTHANS, F. Entrepreneurs as authentic leaders: impact on employees' attitudes. Leadership and organization development journal, Bingley, v. 27, n. 8, p. 646-666, $2006 a$. Disponível em: <http://www.scopus.com/>. Acesso em: 18 dez. 2013.

JENSEN, S. M.; LUTHANS, F. Relationship between entrepreneurs' psychological capital and their authentic leadership. Journal of managerial issues, Pittsburg, v. 18, n. 2, p. 254-273, 2006b. Disponível em: <http://www.scopus.com/>. Acesso em: 18 dez. 2013.

JINGPING, S. Understanding the impact of perceived principal leadership style on teacher commitment. International studies in educational administration, Crediton, v. 32, n. 2, p. 18-31, 2004. Disponível em: <http://search.ebscohost.com>. Acesso em: 18 dez. 2013.

LANZONI, G. M. D.; MEIRELLES, B. H. S. Leadership of the nurse: an integrative literature review. Revista latino-americana de enfermagem, Ribeirão Preto, v. 19, n. 3, p. 651-658, 2011. Disponível em: <http://www.scielo.br/pdf/rlae/v19n3/pt_26.pdf>. Acesso em: 14 out. 2013.

LUTHANS, F. The need for and meaning of positive organizational behavior. Journal of organizational behavior, Oxford, v. 23, n. 6, p. 695-706, 2002. Disponível em: <http://onlinelibrary. wiley.com/doi/10.1002/job.165/pdf>. Acesso em: 13 mar. 2014.

LUTHANS, F.; AVOLIO, B.J. Authentic leadership: a positive developmental approach. In: CAMERON, K. S.; DUTTON, J.E.; QUINN, R. E. (Ed.). Positive organizational scholarship. San Francisco: BarrettKoehler, 2003. p. 241-261.

MENDES, K. S.; SILVEIRA, R. C. C. P.; GALVÃO, C. M. Revisão integrativa: método de pesquisa para a incorporação de evidências na saúde e na enfermagem. Texto contexto - enfermagem, Florianópolis, v. 17, n. 4, p. 758-764, dez. 2008. Disponível em: <http://www.scielo.br/>. Acesso em: 19 nov. 2014.

MITCHIE, S.; GOOTY, J. Values, emotions, and authenticity: will the real leader please stand up? Leadership quarterly, Amsterdam, v. 16, n. 3, p. 441-457, 2005. Disponível em: <www. sciencedirect.com/science/article>. Acesso em: 18 dez. 2013.

NEIDER, L. L.; SCHRIESHEIM, C. A. The Authentic Leadership Inventory (ALI): Development and empirical tests. Leadership quarterly, Amsterdam, v. 22, n. 6, p. 1146-1164, 2011. Disponível em: <www.sciencedirect.com/science/article>. Acesso em: 18 dez. 2013.

NOVICEVIC, M. M. et al. Barnard on conflicts of responsibility. Implications for today's perspectives on transformational and authentic leadership. Management decision, Bingley, v. 43, n. 10, p. 13961409, 2005. Disponível em: <http://www.scopus.com/>. Acesso em: 18 dez. 2013.

NOVICEVIC, M. M. et al. Authentic leadership: a historical perspective. Journal of leadership 
and organizational studies, London, v. 13, n. 1, p. 64-76, 2006. Disponível em: <http://search. ebscohost.com>. Acesso em: 18 dez. 2013.

PRICE, T. L. The ethics of authentic transformational leadership. The leadership quarterly, Amsterdam, v. 14, n. 1, p. 67-81, 2003. Disponível em: <www.sciencedirect.com/science/article>. Acesso em: 18 dez. 2013.

RAHMAN, F.; ABIODULLAH, M.; QURAISHI, U. Authentic leadership for democracy in schools. Academic leadership, Hays, v. 8, n. 2, p. 342-350, 2010. Disponível em: <http://www.scopus.com/>. Acesso em: 18 dez. 2013.

ROTHER, E. T. Editorial: revisão sistemática x revisão narrativa. Acta paulista de enfermagem, São Paulo, v. 20, n. 2, p. v-vi, abr./jun. 2007. Disponível em: <http://www.redalyc.org/articulo. oa? id=307026613004>. Acesso em: 19 nov. 2014.

SELIGMAN, M. Felicidade autêntica: usando a nova psicologia positiva para a realização permanente. Rio de Janeiro: Objetiva, 2004.

SHAMIR, B.; EILAM, G. "What's your story?" A life-stories approach to authentic leadership development. The leadership quarterly, Amsterdam, v. 16, n. 3, p. 395-417, 2005. Disponível em: <www.sciencedirect.com/science/article>. Acesso em: 18 dez. 2013.

SHIREY, M. R. Authentic leaders creating healthy work environments for nursing practice. American journal of critical care, Aliso Viejo, v. 15, n. 3, p. 256-267, May 2006. Disponível em: <http://ajcc. aacnjournals.org/content/15/3/256.full.pdf+html>. Acesso em: 18 dez. 2013.

SOBRAL, F. J. B. A.; GIMBA, R. F. As prioridades axiológicas do líder autêntico: um estudo sobre valores e liderança. Revista de Administração Mackenzie - RAM, São Paulo, v. 13, n. 3, p. 96121, maio/jun. 2012. Disponível em: <http://editorarevistas.mackenzie.br/index.php/RAM/article/ view/4047/3440>. Acesso em: 14 out. 2013.

SOUZA, M. T.; SILVA, M. D.; CARVALHO, R. Revisão integrativa: o que é e como fazer. Einstein, São Paulo, v. 8, n. 1, p. 102-106, 2010. Disponível em: <http://apps.einstein.br/revista/arquivos/ PDF/1134-Einsteinv8n1_p102-106_port.pdf>. Acesso em: 6 dez. 2013.

SPARROWE, R. T. Authentic leadership and the narrative self. The leadership quarterly, Amsterdam, v. 16, n. 3, p. 419-439, 2005. Disponível em: <www.sciencedirect.com/science/article>. Acesso em: 18 dez. 2013.

TATE, B. A longitudinal study of the relationships among self-monitoring, authentic leadership, and perceptions of leadership. Journal of leadership and organizational studies, London, v. 15, n. 1, p. 16-29, 2008. Disponível em: <http://www.scopus.com/>. Acesso em: 18 dez. 2013.

TERRY, R. W. Authentic leadership: courage in action. San Francisco: Jossey-Bass, 1993.

TURNER, J.; MAVIN, S. What can we learn from senior leader narratives? The strutting and fretting of becoming a leader. Leadership and organization development journal, Bingley, v. 29, n. 4, p. 376-391, 2008. Disponível em: <http://www.scopus.com/>. Acesso em: 18 dez. 2013.

WALUMBWA, F. O. et al. Authentic leadership: development and validation of a theory-based measure. Journal of management, London, v. 34, n. 1, p. 89-126, 2008. Disponível em: < http:// jom.sagepub.com/content/34/1/89.full.pdf+html>. Acesso em: 14 out. 2013.

WALUMBWA, F. O. et al. Psychological processes linking authentic leadership to follower behaviors. The leadership quarterly, Amsterdam, v. 21, n. 5, p. 901-914, 2010. Disponível em: <www. sciencedirect.com/science/article>. Acesso em: 18 dez. 2013.

WHITTEMORE, R. Combining evidence in nursing research: methods and implications. Nursing 
research, Baltimore, v. 54, n. 1, p. 56-62, Jan./Feb. 2005. Disponível em: <http://www.scopus.com/>. Acesso em: 19 nov. 2014.

WHITTEMORE, R.; KNAFL, K. The integrative review: updated methodology. Journal of advanced nursing, Malden, v. 52, n. 5, p. 546-553, Dec. 2005. Disponível em: <http://onlinelibrary.wiley.com/ doi/10.1111/j.1365-2648.2005.03621.x/pdf>. Acesso em: 14 nov. 2013.

WONG, C.; CUMMINGS, G. G. Authentic leadership: a new theory for nursing or back to basics? Journal of health, organization and management, Bingley, v. 23, n. 5, p. 522-538, 2009a. Disponível em: <http://www.emeraldinsight.com/doi/pdfplus/10.1108/14777260910984014>. Acesso em: 27 nov. 2014.

WONG, C.; CUMMINGS, G.G. The influence of authentic leadership behaviors on trust and work outcomes of health care staff. Journal of leadership studies, Malden, v. 3, n. 2, p. 6-23, 2009b. Disponível em: <http://search.ebscohost.com>. Acesso em: 18 dez. 2014.

WONG, C.; GIALLONARDO, L. M. Authentic leadership and nurse-assessed adverse patient outcomes. Journal of nursing management, Chichester, v. 21, n. 5, p. 740-752, 2013. Disponível em: <http://onlinelibrary.wiley.com/doi/10.1111/jonm.12075/pdf>. Acesso em: 18 dez. 2013.

WONG, C.; LASCHINGER, H. K. S.; CUMMINGS, G. G. Authentic leadership and nurses' voice behaviour and perceptions of care quality. Journal of nursing management, Chichester, v. 18, n. 8, p. 889900, 2010. Disponível em: <http://onlinelibrary.wiley.com/doi/10.1111/j.1365-2834.2010.01113.x/ pdf $>$. Acesso em: 18 dez. 2013.

YAMMARINO, F. J. et al. Authentic leadership and positive organizational behavior: a meso, multilevel perspective. The leadership quarterly, Amsterdam, v. 19, n. 6, p. 693-707, 2008. Disponível em: <www.sciencedirect.com/science/article>. Acesso em: 18 dez. 2013.

ZHENG, X. et al. Ethical leadership in Chinese organizations: developing a scale. Frontiers of business research in China, Leiden, v. 5, n. 2, p. 179-198, 2011. Disponível em: <http://www. scopus.com/>. Acesso em: 18 dez. 2013. 